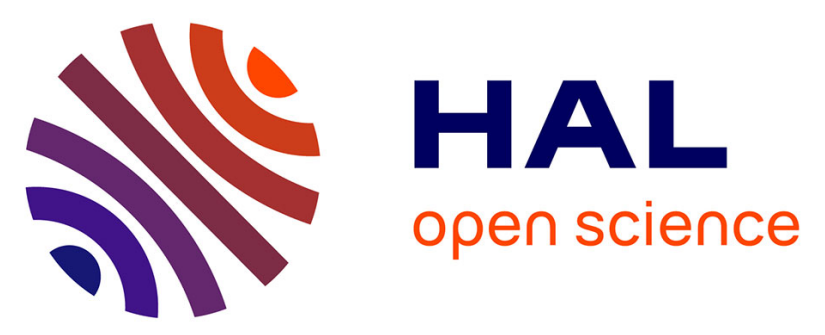

\title{
Influence of Lode angle on modelling of void closure in hot metal forming processes
}

Abdelouahed Chbihi, Pierre-Olivier Bouchard, Marc Bernacki, Daniel Pino Muñoz

\section{- To cite this version:}

Abdelouahed Chbihi, Pierre-Olivier Bouchard, Marc Bernacki, Daniel Pino Muñoz. Influence of Lode angle on modelling of void closure in hot metal forming processes. Finite Elements in Analysis and Design, 2017, 126, pp.13 - 25. 10.1016/j.finel.2016.11.008 . hal-01424267

HAL Id: hal-01424267

https://hal-mines-paristech.archives-ouvertes.fr/hal-01424267

Submitted on 13 Jun 2018

HAL is a multi-disciplinary open access archive for the deposit and dissemination of scientific research documents, whether they are published or not. The documents may come from teaching and research institutions in France or abroad, or from public or private research centers.
L'archive ouverte pluridisciplinaire HAL, est destinée au dépôt et à la diffusion de documents scientifiques de niveau recherche, publiés ou non, émanant des établissements d'enseignement et de recherche français ou étrangers, des laboratoires publics ou privés. 


\title{
Influence of Lode angle on modelling of void closure in hot metal forming processes
}

\author{
A. Chbihi*, P.-O. Bouchard, M. Bernacki, D. Pino Muñoz
}

Mines ParisTech,CEMEF - Centre de Misee Forme des Matériaux, CNRS UMR 7635 CS 10207,1 rue Claude Daunesse, 06904 Sophia Antipolis cedex, France

*Corresponding author. Tel.: +33493678924

E-mail address: abdelouahed.chbihi@mines-paristech.fr

\begin{abstract}
The presence of voids after casting processes of large metal workpieces require the use of adapted hot metal forming processes to deliver sound products. Yet, there is at present a lack of knowledge regarding void closure mechanisms and there is no reliable model that can accurately predict void closure. The main aim of this work is to develop a highly accurate mean field model able to predict the evolution of the voids volume after each forming stage. This proposed model is accounting for both stress triaxiality ratio $T_{x}$ and Lode angle $\theta$ in order to override the hypothesis of axisymmetric loading, which is generally considered in the existing models handling void closure in the literature. Based on an advanced multiscale approach, this model also accounts for voids shape and orientation. An optimization method, using a database of explicit RVE simulations, is developed in order to calibrate the new proposed model. Several void morphological parameters and an industrial range of mechanical loading parameters are analyzed regarding void closure. The proposed mean field model is validated by comparison with explicit full field simulations and
\end{abstract}


with a recent pre-existing mean field model, named Cicaporo1 model hereafter (Saby et al., 2014b). In comparison with Cicaporo1, the new model, named Cicaporo2 hereafter, uses less constants and is more accurate.

\section{Introduction}

Microvoids are frequently detected during large ingots production, which decrease the material quality thereafter. In the industry, hot forming processes are generally used to close these voids and end up with sound products. To insure that the total closure is achieved, the use of 3D X-ray analyses is not possible on such large ingots. Ultrasonic analysis is an alternative solution to detect defects on final products, but its accuracy is quite low and it does not help in finding solutions to close them. Therefore, mathematical models are useful to predict void volume evolution and provide an estimation of void closure for given applied plastic strain under given stress states.

To deal with this industrial issue, numerous models were defined in the literature using two principal approaches: macroscopical approach and micro-analytical approach. The first approach considers a full-field explicit description of an entire workpiece containing explicit voids. Using adequate numerical features, the description may be accurate for studying void closure in real processes according to a given case study. In micro-analytical analyses, a single void in an infinite incompressible matrix is considered. The matrix is usually considered viscoplastic and is defined by a power law type. The evolution of the initial void is studied with respect to various mechanical parameters, for different types of material. A large number of studies consider this approach (Zhang et al., 2009), (Saby et al., 2014a) and (Feng and Cui, 2015). Voids are typically assumed to be spherical or cylindrical. Most studies do not consider any change of shape during strain and the resulting equations are often inappropriate for large deformation (Budiansky et al., 1982), (Lee and Mear, 1994) and (Zhang et al., 2009). 
However, both approaches have significant limitations in industrial applications. For instance, macroscopic approaches undermine difficulties to take into account, in the same simulations, void-scale characteristics and process-scale parameters. This can lead to the use of heavy meshes, case-dependent description/results and high numerical cost. Regarding micro-analytical approaches, assumptions made for void geometries, loading conditions and material laws are usually too strong for real industrial processes modelling.

It is worth mentionning that the elimination of internal voids is achieved in two principal stages: closure of void volume until the contact between faces is reached and the subsequent bonding of the contact surfaces under sufficiently high temperature and compressive stresses (Park and Yang, 1996). The present work concerns only the void closure stage.

(Ståhlberg et al., 1980) presented a micro-analytical criterion for void closure. They considered square and circular voids in a rigid perfectly plastic material. The proposed model defines the necessary reduction $R_{c}$ to completely close the void to be proportional to the square root of its initial volume $V_{0}$. Experiments were carried out in order to validate the theoretical model and an agreement was found. (Tanaka et al., 1986) suggested that the integral $Q$ of stress triaxiality ratio $T_{x}$ over equivalent strain $\bar{\epsilon}\left(Q=\int T_{x} d \bar{\epsilon}\right)$ was a better way to predict void closure. They developed an empirical void closure parameter based on $Q$ and using linear regression to get model constants. Based on the work of Lasne, (Saby, 2013) introduced the Stress Triaxiality Based (STB) model which is implemented in the finite element software FORGE ${ }^{\circledR}$. The STB model states that the void volume prediction could be computed by the integration of the stress triaxiality $T_{x}$ multiplied by a proportionality coefficient, identified by simulations of compression on a spherical void, over the equivalent strain. (Budiansky et al., 1982) proposed an analytical solution which takes into account the influence of the stress triaxiality $T_{x}$ and introduces a material parameter $m$ and considered voids as spherical during the whole strain. Based on this solution (Zhang et al., 2009) proposed a semi-empirical solution to predict 
void closure adding the influence of the void shape evolution. (Lee and Mear, 1994) stated that the non-spherical form of void in the beginning of deformation can significantly influence the evolution of void closure. In fact, real voids exhibit complex forms with significant tortuosity, which makes a sphere far from real voids shape. Therefore, by using ellipsoidal voids, which are closer to reality, the orientation and elongation of voids could also be studied. Recently, (Feng and Cui, 2015) studied the evolution of dilute ellipsoidal void in power law viscous material under triaxial loading conditions. A semi-analytical expression was defined to predict void closure for large compressive strain. The model accounts for the void's shape to characterize the pores and assumes that the void's orientation is parallel to the principle stress, which is not in agreement with experimental observations. (Saby et al., 2015) used a mesoscale method to develop a model based on a large campaign of simulations on Representative Volume Elements (RVE). This is the only model, to the authors' knowledge, that accounts for the geometry of voids (orientation and form) in addition to mechanical loading parameters (stress triaxiality ratio $T_{x}$ and equivalent strain $\bar{\epsilon}$. Several configurations of voids were tested in a large range of mechanical loading conditions corresponding to industrial applications. This model (Cicaporo1) presented a great accuracy compared to explicit simulations of real industrial processes (Saby et al., 2014a). Nevertheless, one of its limitations lies on the assumption of axisymmetric loading. Indeed, all void closure prediction models existing in the literature consider this hypothesis, for which loading conditions are defined using only stress triaxiality. However, this parameter does not allow to define a unique stress state. A detailed review of existing models can be found in (Saby et al., 2014b).

Therefore, the aim of the present work is to propose an enhanced model based on an improved and more accurate description of the stress state in the material. This is achieved by involving the three stress invariants.

Within the context of material damage and failure analysis, many authors have introduced the Lode angle $\theta$, which is a function of the third invariant of deviatoric 
stress tensor. (Danas and Castañeda, 2012) modelled the damage of porous elastoplastic materials by two approaches according to the state of the stress triaxiality. They proved that for triaxial tensile cases, the Lode angle appears to have no effect on the developed model. However, it seems to be an influencing factor for triaxial compressive cases. (Cao et al., 2014) studied the calibration of ductile damage models for high strength steels using microtomography. They confirmed that the use of the Lode angle is a good way to represent the stress state for low or negative stress triaxiality conditions. Other works realized by (Keshavarz et al., 2014), (Xue and Wierzbicki, 2008) and (Mirone and Corallo, 2010) confirmed that the combination of the stress triaxiality ratio $T_{x}$ and Lode angle $\theta$ was necessary to define the stress state accurately.

In this paper, a new void closure model is proposed to improve the prediction accuracy by eliminating the axisymmetric loading hypothesis. The calibration of this model is achieved by using a database of explicit RVE simulations. In the following section, the mesoscale method used to perform explicit simulations is presented. Several configurations of voids and mechanical loading conditions are considered in order to study their influence on void closure. The way of defining boundary conditions on the RVE for given stress triaxiality and Lode angle is detailed. The third section concerns the influence of specific parameters on void closure: void orientation and form as geometry parameters and applied stress triaxiality and Lode angle as mechanical loading parameters. The fourth section is dedicated to the development of the calibration methodology. This methodology is based on an optimization algorithm that enables the identification of the void closure model parameters according to a large number of RVE explicit simulations. This optimization approach also enables to change the void closure analytical function and to proceed to a new calibration in a reduced time. In the fifth section, results of the new model are presented and compared to RVE explicit simulations and Cicaporo1 model predictions. Last section is dedicated to conclusions and perspectives 


\section{Mesoscale approach and boundary conditions}

In this section, a short description of the mesoscale approach used to perform explicit simulations is presented. For more details, the reader can refer to (Saby et al., 2013). Boundary conditions are also detailed in order to take into account the effect of Lode angle $\theta$ in addition to stress triaxiality ratio $T_{x}$.

\subsection{Representative Volume Element (RVE)}

In this work, a mesoscale method is used to perform full-field simulations. In fact, void dimensions are assumed very small with respect to the workpiece dimensions. Several works considerd a small void/billet ratio: 0.01 to 0.06 in (Kakimoto et al., 2010), 0.05 in (Wallerö, 1985) and 0.06 in (Wang et al., 1996). From this condition, two main assumptions can be made: a) the presence of voids has no effect on macroscopic deformation and b) the thermo-mechanical fields that are obtained from the macroscopic scale are considered as locally homogeneous and can be used as remote boundary conditions at RVE scale (Saby, 2013) and (Saby et al., 2013). The objective is then to perform multiple explicit simulations with stress states representative of industrial conditions (stress triaxiality ratio $T_{x}$ and Lode angle $\theta$ ). The finite element (FE) simulations were performed using the software Forge ${ }^{\circledR}$ NxT1.0. The void were represented by an ellipsoid of main dimensions $\left(r_{1}, r_{2}, r_{3}\right)$ located at the center of the RVE. A convergence study has been carried out in order to determine the dimensions of the RVE and the optimal mesh size. The RVE dimensions $\left(d_{1}, d_{2}, d_{3}\right)$ are set as four times the ellipsoid initial radii. The maximum mesh size in the RVE is $0.5 \mathrm{~mm}$ while $r_{1}, r_{2}, r_{3}$ vary between $1 \mathrm{~mm}$ and $4 m m$. A mixed velocity-pressure formulation with $P_{1}^{+} / P_{1}$ tetrahedral elements and local refinement are used for an accurate description of void closure.

The viscoplastic material is defined by a Hansel-Spittel law. The equivalent stress is then expressed as:

$$
\bar{\sigma}=A(\bar{\epsilon}, T) * \dot{\bar{\epsilon}}^{m}
$$


with

$$
A(\bar{\epsilon}, T)=K_{0} T^{m_{1}}\left(\bar{\epsilon}+\epsilon_{0}\right)^{n} e^{m_{4} /\left(\bar{\epsilon}+\epsilon_{0}\right)},
$$

where $\bar{\epsilon}$ is the equivalent strain, $T$ is the temperature, $m$ is the strain rate sensitivity, $K_{0}$ is the material consistency, $m_{1}$ the temperature sensitivity, $\left(n, m_{4}\right)$ the hardening and softening coefficients, respectively, and $\epsilon_{0}$ is a regularization term.

\subsection{Boundary conditions}

\subsubsection{Axisymmetric loading hypothesis}

Most of void volume prediction models in the literature (Tanaka et al., 1986), (Zhang et al., 2009), (Saby et al., 2014a) only take into account the equivalent strain $\bar{\epsilon}$ and the stress triaxiality ratio $T_{x}$, and consider axisymmetric loading conditions. At the RVE scale, the industrial boundary conditions are represented by a velocity applied on the top of the RVE (to obtain a given strain rate) and two identical stresses are applied on the two lateral sides of the RVE $\left(\sigma_{1}=\sigma_{2}\right)$. Three symmetry planes $\left(S_{1}, S_{2}, S_{3}\right)$ are set in the remaining sides (with green, red and blue colors), as illustrated in Figure 1. The velocity $V_{z}$ applied on the top surface is defined so as to apply a given constant strain rate value $\dot{\epsilon}_{z z}$ on the RVE. $V_{z}(t)$ is then defined as $-\dot{\epsilon}_{z z} D_{z}(t)$ with $D_{z}(t)$ the current RVE height. It is worth mentioning that the equivalent strain rate has a second order influence on void closure for our materials and thermomechanical conditions of interest (Saby et al., 2015), therefore, it will not be considered as a parameter of the new model. So a value $\dot{\epsilon}_{z z}=1 \mathrm{~s}^{-1}$ is used for all the explicit simulations on RVE.

In order to reproduce real industrial conditions, it is important to address more general loading conditions and then a new approach is proposed in the following. For such general stress state, it can be shown that the stress triaxiality ratio is not sufficient to describe the stress state in a unique way.

Indeed, different stress fields can lead to the same state of triaxiality $T_{x}$ and equivalent strain rate $\dot{\bar{\epsilon}}$. Figure 2 illustrates two stress states, which can be applied 

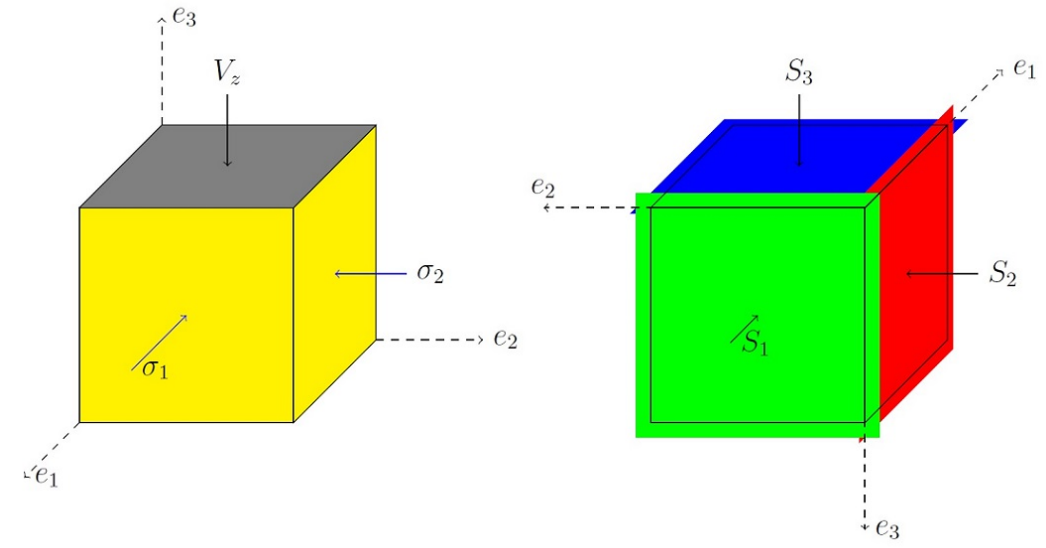

Figure 1: The boundary conditions set to the RVE in the canonical basis $\left(\overrightarrow{e_{1}}, \overrightarrow{e_{2}}, \overrightarrow{e_{3}}\right)$.

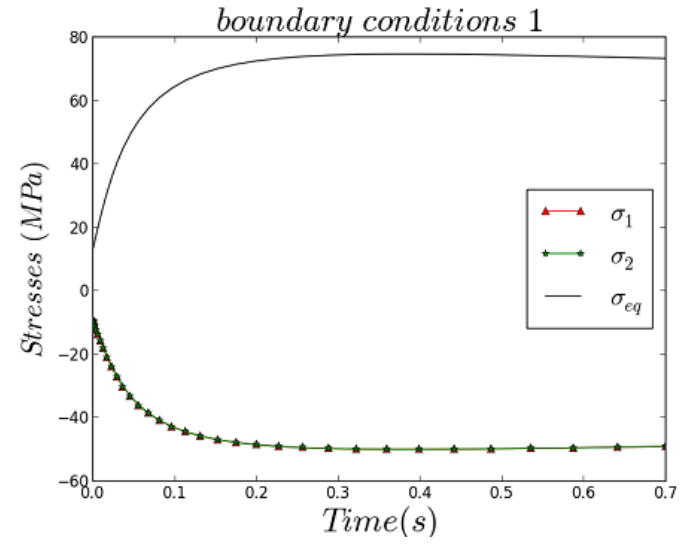

(a)

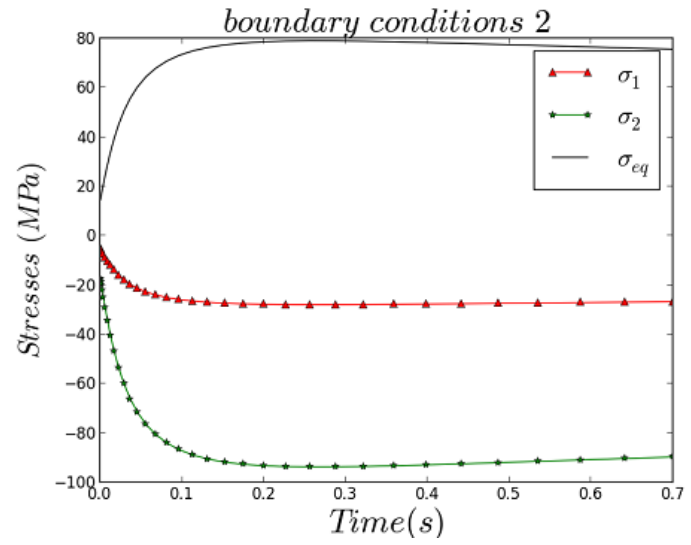

(b)

Figure 2: Different stress states for the same values $\dot{\epsilon}_{z z}=-1 s^{-1}$ and $T_{x}=-1$.

to the RVE as boundary conditions, as presented in Figure 1, and which lead to the values $\dot{\epsilon}_{z z}=-1 s^{-1}$ and $T_{x}=-1$. Note that the equivalent stress $\bar{\sigma}$ is the same in both cases. In the first case (Figure 2a) the equivalent stress $\bar{\sigma}$ is plotted with black solid line while $\sigma_{1}$ and $\sigma_{2}$ are plotted with red triangles and green stars respectively. The evolutions of $\sigma_{1}$ and $\sigma_{2}$ are identical which means that it corresponds to axisymmetric loading. In the second case (Figure $2 \mathrm{~b}$ ), the stresses $\sigma_{1}$ and $\sigma_{2}$ are different even if the stress triaxiality ratio is the same $\left(T_{x}=-1\right)$, this case does not correspond to axisymmetric loading. Therefore, a third parameter should be added in order to represent the stress state in a unique way. 


\subsubsection{Non-axisymmetric loading conditions and Lode angle $\theta$}

In this work, the axisymmetric hypothesis is removed. The Lode angle $\theta\left(0 \leq \theta \leq \frac{\pi}{3}\right)$, which is linked to the third invariant of deviatoric stress tensor (see Eq. 5) by Eq. 7 , is added. The stress state is characterized by the 3 eigenvalues of the stress tensor $\left(\sigma_{1}, \sigma_{2}, \sigma_{3}\right)$ and can also be expressed by the first invariant of the stress tensor $\left(I_{1}\right)$ and by the second and third invariants of the deviatoric stress tensor $\left(J_{2}\right.$ and $\left.J_{3}\right)$, respectively:

$$
\begin{gathered}
\sigma_{h}=\frac{1}{3}\left(\sigma_{1}+\sigma_{2}+\sigma_{3}\right)=\frac{I_{1}}{3} \\
\bar{\sigma}=\sqrt{\frac{1}{2}\left[\left(\sigma_{1}-\sigma_{2}\right)^{2}+\left(\sigma_{2}-\sigma_{3}\right)^{2}+\left(\sigma_{3}-\sigma_{1}\right)^{2}\right]}=\sqrt{3 J_{2}} \\
r=\sqrt[3]{\frac{27}{2}\left(\sigma_{1}-\sigma_{h}\right)\left(\sigma_{2}-\sigma_{h}\right)\left(\sigma_{3}-\sigma_{h}\right)}=\sqrt[3]{\left(\frac{27}{2} J_{3}\right)} .
\end{gathered}
$$

Then, the stress triaxiality is obtained by the ratio between the hydrostatic stress $\sigma_{h}$ and the equivalent stress $\bar{\sigma}$ (Eq. 6), while the Lode angle $\theta$ is expressed by Eq. (7):

$$
\begin{gathered}
T_{x}=\frac{\sigma_{h}}{\bar{\sigma}}=\frac{\sigma_{1}+\sigma_{2}+\sigma_{3}}{3 \bar{\sigma}}=\frac{I_{1}}{3 \sqrt{3 J_{2}}}, \\
\cos 3 \theta=\left(\frac{r}{\bar{\sigma}}\right)^{3} .
\end{gathered}
$$

For a better understanding of the meaning of Lode angle $\theta$, reader can consult (Cao et al., 2014) where more details are presented with a graphical scheme.

A simplified expression of the Lode angle (Eq. 7) was proposed by (Lode, 1926) introducing the Lode parameter $\mu$ in Eq. (9) $(-1 \leq \mu \leq 1)$. This Lode parameter $\mu$ is related to the Lode angle $\theta$ by (Eq. 8). It is worth mentionning that the use of the Lode parameter requests that $\sigma_{1} \geq \sigma_{2} \geq \sigma_{3}$, with $\sigma_{1} \neq \sigma_{3}$ :

$$
\begin{gathered}
\mu=\sqrt{3} \cdot \tan \left(\theta-\frac{\pi}{6}\right) . \\
\mu=\frac{2 \sigma_{2}-\sigma_{1}-\sigma_{3}}{\sigma_{1}-\sigma_{3}} .
\end{gathered}
$$

The stress state is then defined by $\left(T_{x}, \bar{\sigma}, \mu\right)$. 


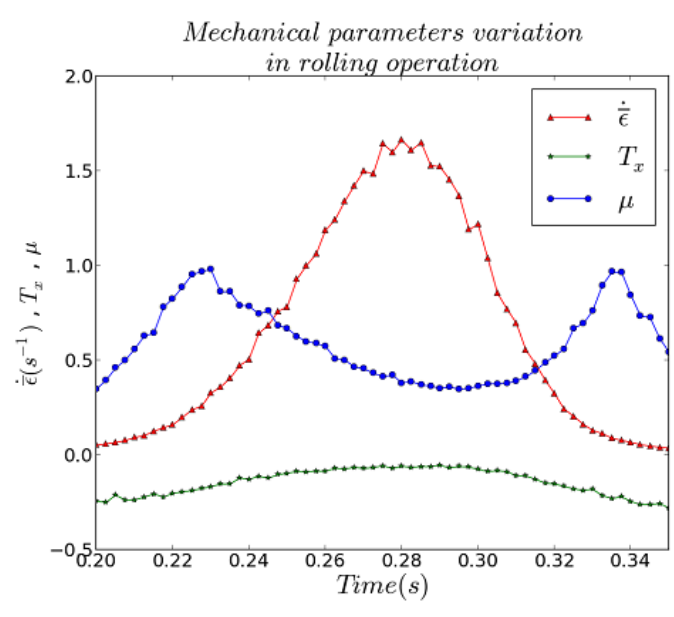

(a) Rolling case

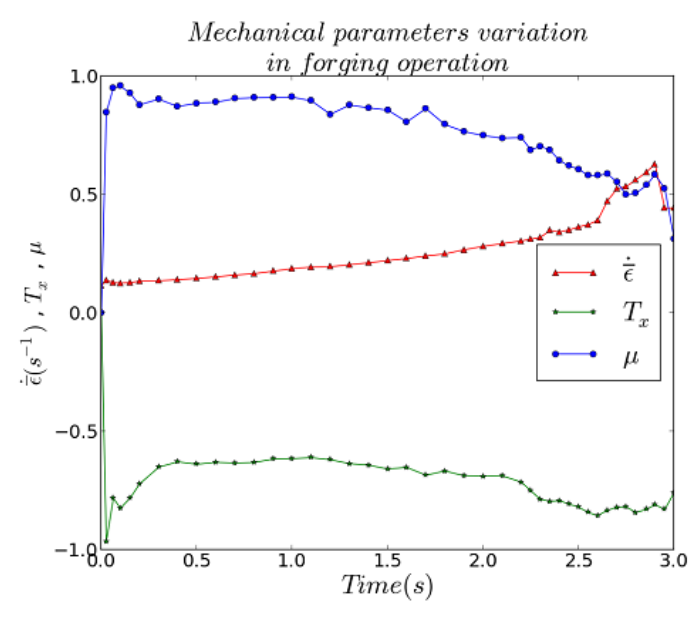

(b) Forging case

Figure 3: Variation of stress state and strain rate during industrial processes.

\subsubsection{Industrial loading conditions}

In order to perform the explicit simulations, the range of mechanical loading conditions observed in industrial applications needs to be determined. Therefore, simulations of industrial processes are performed in order to analyze the variation equivalent strain rate $\dot{\bar{\epsilon}}$ (red line), stress triaxiality ratio $T_{x}$ (green line) and Lode parameter $\mu$ (blue line); which are plotted versus equivalent strain time on figure 3. Rolling and forging processes are modelled and a sensor is located at the ingot's center, which is the place where voids are more likely to exist after casting. This sensor enables to record the mechanical loading conditions during the process. The variation of mechanical loading parameters is plotted in the range of interest, which corresponds to significant strain rate $\dot{\epsilon}_{z z} \geq 0$ or $0.17 \mathrm{~s} \leq$ Time $\leq 0.37 \mathrm{~s}$. In this range, $T_{x}$ varies between -0.4 and -0.1 while $\mu$ ranges from 0.1 and 1 (Figure $3 \mathrm{a})$. The same procedure is done for the case of forging (Figure 3b). The strain rate is non zero during the whole process. The Lode parameter varies between 0.5 and 1 . Therefore, the axisymmetric loading hypothesis $(\mu=1)$ is not consistent with mechanical loading conditions representative of industrial processes.

In fact, as it can be seen from Figure 3, it is difficult to reproduce exactly the stress state variation corresponding to a given forming process. Consequently, the 
methodology chosen is the following: boundary conditions are iteratively modified to prescribe constant values of strain rate $\dot{\bar{\epsilon}}$, stress triaxiality ratio $T_{x}$ and Lode parameter $\mu$ during RVE simulations up to complete void closure, in such a way that the loading ranges are covered. This method allows to generate a strain and stress states which could represent a range of mechanical loading at the RVE scale always found in industrial processes as it is presented in Figure 3. In this figure only one case of rolling and forging are presented, however more industrial processes are studied and the same conculsions were reached. The efficiency of this method to control the evolution of loading conditions is shown in (Saby et al., 2013).

In the case of axisymmetric loading hypothesis, the applied stresses on the RVE lateral sides are easy to compute. It needs the resolution of a system of two linear equations (Eq. 6) and (Eq. 4). In addition, the equivalent strain rate $\dot{\bar{\epsilon}}$ is equal to the strain rate in the deformation direction $\dot{\epsilon}_{z z}$. However, by removing this hypothesis, the resolution becomes more complicated as the equations are in their general forms. The equivalent strain rate $\dot{\bar{\epsilon}}$ is now expressed by Eq.(10), so that the system of equations is nonlinear. The idea is to find the tractions $\left(\sigma_{x x}, \sigma_{y y}\right)$ and the velocity $V_{z}$ that should be applied to the RVE in such a way that the desired Triaxiality $T_{x}$, Lode's Angle $\mu$ and strain rate $\dot{\epsilon}_{z z}$ are obtained. Since the material behavior is not linear, then this is done by solving the set of equations (4), (6) and (9). This equations set could be resolved by analytical development, optimization methods or other opproaches. In this work, an optimization algorithm has been developed to compute the boundary conditions to set on the RVE with respect to the constitutive law (Eq. 1), the equivalent strain rate (Eq. 10) and volume conservation (Eq. 11).

$$
\begin{gathered}
\dot{\bar{\epsilon}}=\sqrt{\frac{2}{3}\left(\dot{\epsilon}_{1}^{2}+\dot{\epsilon}_{2}^{2}+\dot{\epsilon}_{3}^{2}\right)}, \\
\dot{\epsilon}_{1}+\dot{\epsilon}_{2}+\dot{\epsilon}_{3}=0
\end{gathered}
$$

Note that this optimization concerns only the computing of the boundary conditions to set to RVE. Another optimization handling the identification of the mean- 
field model's parameters will be discussed later.

\subsection{Void Morphology}

To define the initial topology of the void during RVE explicit simulations, three orientations $\left(\alpha_{1}, \alpha_{2}, \alpha_{3}\right)$ regarding the canonical basis $\left(\overrightarrow{e_{1}}, \overrightarrow{e_{2}}, \overrightarrow{e_{3}}\right)=(\mathrm{x}, \mathrm{y}, \mathrm{z})$ and corresponding dimensions $\left(r_{1}, r_{2}, r_{3}\right)$ are defined as shown in Figure 4. However, in order to study the influence of the void's topology on void closure, dimensionless parameters are prefered. The orientation factors $\left(p_{1}, p_{2}, p_{3}\right)$ are defining the ellipsoid orientation. $p_{i}$ are defined by the vectorial product between the main direction $\overrightarrow{e_{i}}$ of the void and the loading direction $\overrightarrow{e_{L}}$ as expressed in Eq. (12). The loading direction $\overrightarrow{e_{L}}$ is computed using a combination of the principal stresses and the three main directions of the canonical basis $\overrightarrow{e_{i}}$ by the expression in Eq. (13).

$$
\begin{gathered}
p_{i}=\overrightarrow{e_{L}} \otimes \overrightarrow{e_{i}}, \\
\overrightarrow{e_{L}}=\frac{\sigma_{1} \overrightarrow{e_{1}}+\sigma_{2} \overrightarrow{e_{2}}+\sigma_{3} \overrightarrow{e_{3}}}{\left\|\sigma_{1} \overrightarrow{e_{1}}+\sigma_{2} \overrightarrow{e_{2}}+\sigma_{3} \overrightarrow{e_{3}}\right\|},
\end{gathered}
$$

Finally, shape factors $\left(\gamma_{1}, \gamma_{2}, \gamma_{3}\right)$ are defined thanks to Eq. (14) with $V_{0}$ the initial volume of the ellipsoid. Note that the geometrical description of the void is given bi $\alpha_{i}$ and $r_{i}$, while $p_{i}$ and $\gamma_{i}$ are used as input for the void closure prediction model.

$$
\gamma_{i}=\frac{\sqrt[3]{V_{0}}}{r_{i}}
$$

\section{Influence of Lode parameter on void closure}

After describing the methodology used to define the RVE boundary conditions and the range of stress states observed in industrial configurations, the influence of Lode parameter $\mu$ on void closure at RVE scale is studied.

As it was observed in the variation of mechanical loadings in industrial processes (Figure 3 ), the range of variation of Lode parameter is $[0,1]$. However, this study 

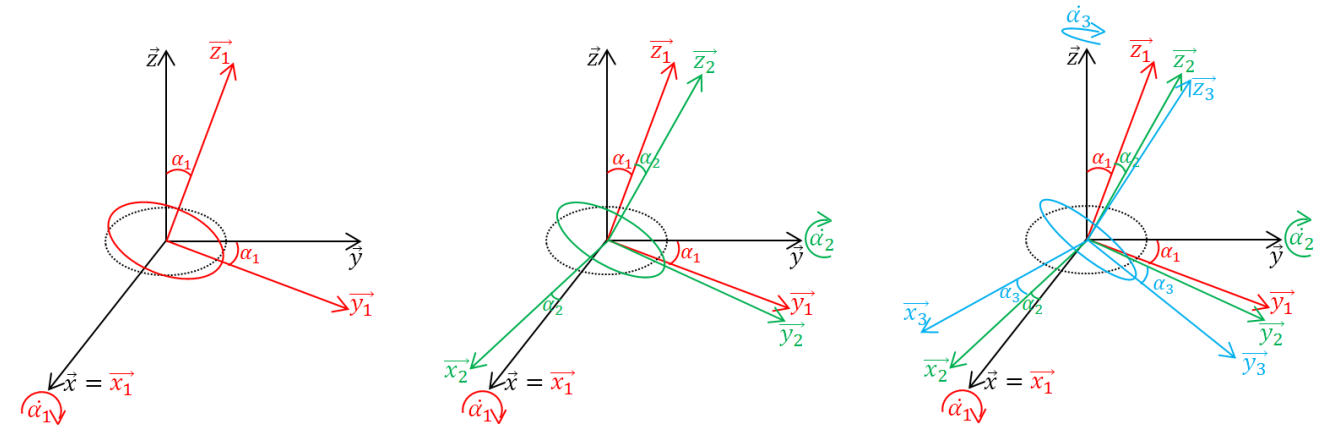

Figure 4: Topological definition of ellipsoids with Euler Angles: $r_{i}=$ $(2.36,1.62,1.34), \alpha_{i}=\left(45^{\circ}, 45^{\circ}, 0^{\circ}\right)$.

could be applied for the whole range of definition of Lode parameter $\mu \in[-1,1]$ as this is its theoritical range of variation. So the range $[-0.5,1]$ will be considered in hereafter.

In this section, only one ellipsoid geometry is presented in order to illustrate the influence of the Lode parameter on void closure. Note that this geometry is chosen randomly in the dimensions variation range. It is used as presented in Figure 4 with $\left(\alpha_{1}, \alpha_{2}, \alpha_{3}\right)=\left(45^{\circ}, 45^{\circ}, 0^{\circ}\right)$ and $\left(r_{1}, r_{2}, r_{3}\right)=(2.36,1.62,1.34)$. Different states of stress triaxiality ratio $T_{x} \in\{-1,-0.66,-0.33\}$ and obviously different values of Lode parameter $\{-0.5,0,0.5,1\}$ are used in order to evaluate its influence on volume decrease. Figure 5 shows the results of a set of simulations for the presented ellipsoid and for each considered stress state, where void closure evolution $\left(V / V_{0}\right)$ is plotted versus equivalent strain $(\bar{\epsilon})$. The cases $\mu=1, \mu=0.5, \mu=0$ and $\mu=-0.5$ are plotted in salmon, blue, cyan and red, respectively. The straight line $y=0.2$ corresponds to a threshold value of void closure. In fact, during last stages of closure, the tortuosity of real voids, in industrial processes, decelerates the void closure due to the contact between the void's internal faces, especially in the range [0,0.2] (Saby, 2013) and (Zhang et al., 2009). Consequently, hereafter, only the range $[0.2,1]$ of void closure $V / V_{0}$ is considered as realistic and discussed. The gap of strain $\Delta \bar{\epsilon}$, defined by Eq. (15), presents the difference of strain between the case $\mu=1$ and the case $\mu=-0.5$ at the threshold value of void closure $V / V_{0}=0.2$. 


$$
\Delta \bar{\epsilon}=\left|\bar{\epsilon}_{\mu=1}-\bar{\epsilon}_{\mu=-0.5}\right|
$$

In Figure 5a, where $T_{x}=-1$, which is a favorable case for closure (Saby et al., 2015), the axisymmetric loading condition $\mu=1$ is also favorable for void closure. The strain difference is really small $(\Delta \bar{\epsilon}=0.05)$ which means almost no influence of the Lode parameter on void closure. Then, looking at Figure 5b, where $T_{x}=-0.66$, the gap becomes more important with $\Delta \bar{\epsilon}=0.1$. The same behavior, concerning the influence of $\mu$ on void closure, is noted again. So that, the gap is still increasing for the case $T_{x}=-0.33$ (Figure $5 \mathrm{c}$ ) with $\Delta \bar{\epsilon}=0.24$. In the last case, $T_{x}=0$, an unfavorable case for closure, the gap is even more important (Figure 5d). In fact, $\Delta \bar{\epsilon}$ cannot be defined at the threshold value of closure because the void is not completely closed due to its orientation and the mechanical loading condition that represents a generalized shear. However, at the last increment of closure $\left(V / V_{0}=0.4\right), \Delta \bar{\epsilon}=0.48$.

Considering the trend observed in Figure 5, two results could be noted: on the one hand, Lode parameter $\mu$ has a significant influence on void closure, especially when $T_{x}$ is close to 0 . On the other hand, the higher is $\mu$, the faster is the void closure, regardless of the type of geometry neither void geometry. A comparison between different void geometry and orientation is given in Figure 6. This bevavior is respected even for a sphere with $r=2$ (Figure 6a), an oblate ellipsoid with $r_{i}=(2,2,1)$ and $\alpha_{i}=(0,0,0)$ (Figure $6 \mathrm{~b}$ )or a prolate ellipsoidal void with $r_{i}=$ $(2,1,1)$ and $\alpha_{i}=(45,45,0)$ (Figure 6c). Consequently, the axisymmetric hypothesis used in the literature represents the most favorable case for the void closure study. Therefore, it seems quite obvious that the use of this new parameter $(\mu)$ must be taken into account in order to improve the accuracy of prediction models used in this field of research.

Therefore, a large campaign of explicit simulations on RVE needs to be carried out in order to cover the prescribed ranges for all parameters: $T_{x} \in[-1,-0.33]$, $\mu \in[-0.5,1], \alpha_{i} \in[0, \pi]$ and $r_{i} \in[1,3]$. However, if only 5 values are considered for each parameter, nearly 16000 cases should be carried out, which is too expensive 


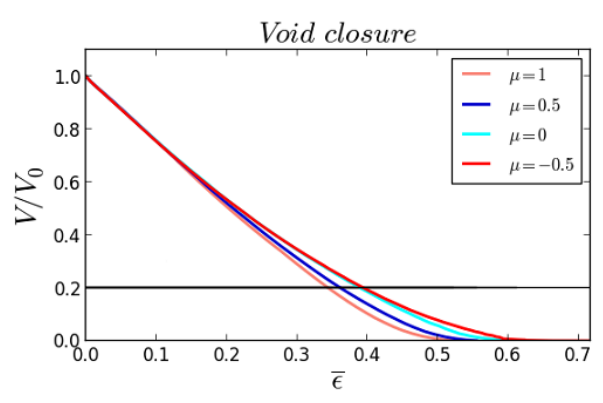

(a) $T_{x}=-1$

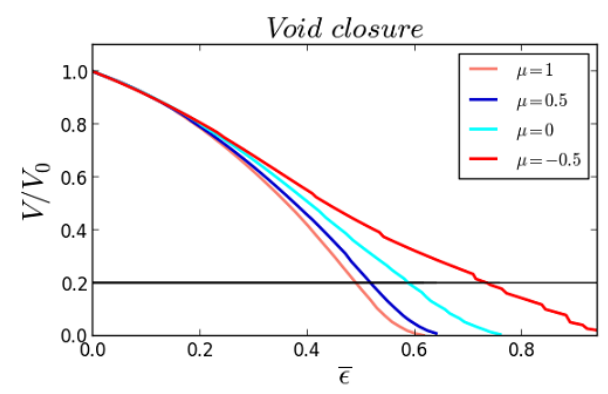

(c) $T_{x}=-0.33$

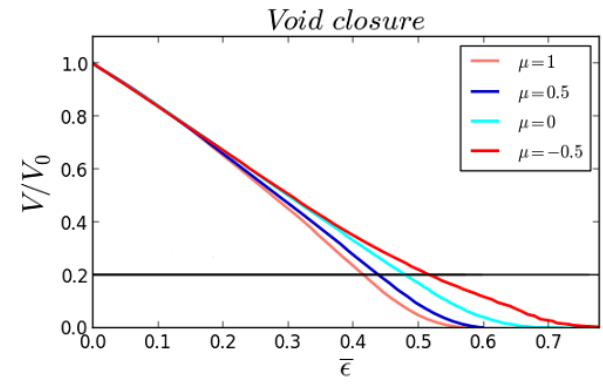

(b) $T_{x}=-0.66$

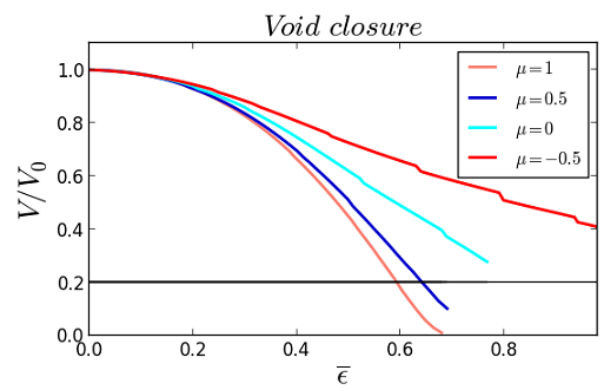

(d) $T_{x}=0$

Figure 5: Influence of Lode parameter $\mu$ on void closure for 4 different stress triaxiality.

in term of computation time. Thus, a reduced number of cases is used here as following:

- 4 values of $\mu:-0.5,0,0.5,1$

- 3 values of $T_{x}:-1,-0.66,-0.33$

- 4 shapes of ellipsoids $\left(r_{1}, r_{2}, r_{3}\right):(2,2,1),(2,1,1),(2,1.5,1),(2.36,1.62,1.34)$

- 5 orientations per axis: $0^{\circ}, 45^{\circ}, 90^{\circ}, 135^{\circ}, 180^{\circ}$ (All similar cases are eliminated).

A database is used to store all these configurations (695), and it can be easily enhanced if new configurations are needed to enrich the model. More details are presented in section 4 . 


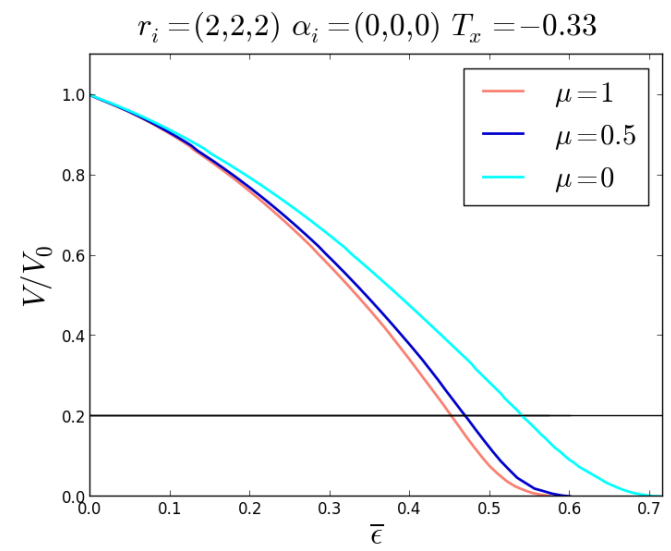

(a) Sphere

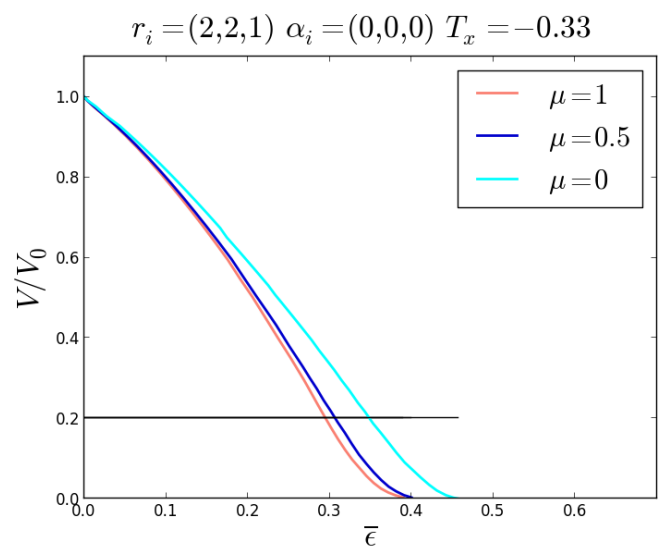

(b) Oblate ellipsoid

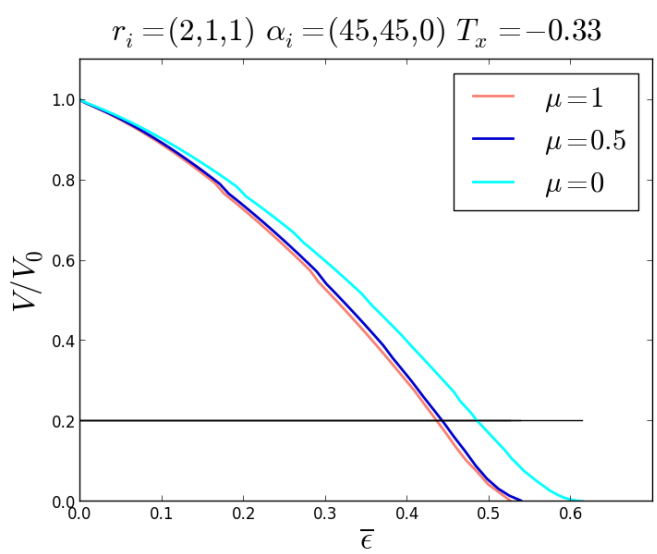

(c) prolate ellipsoid

Figure 6: Influence of Lode parameter $\mu$ on void closure for different void geometry.

\section{Optimization method and model construction}

In the previous section, the influence of the Lode parameter $\mu$ was presented. The new prediction model (Cicaporo2), which includes the Lode parameter $\mu$, is now detailed. In addition, the optimization method required for the model calibration is also introduced. It is worth mentioning that this model takes into account equivalent strain $\bar{\epsilon}$, stress triaxiality ratio $T_{x}$, Lode parameter $\mu$, orientation factors $p_{i}$ (Eq. (12)) and void's shape factors $\gamma_{i}$ (Eq. (14)). This work will not include material parameters effect, as it was shown by (Saby et al., 2015) they have a second order influence on void closure for our material and thermomechanical conditions of interest. 


\subsection{The choice of the analytical form}

To develop a mean field prediction model, (Saby et al., 2015) and (Zhang et al., 2009) used nonlinear regression to fit models constants based on RVE FE simulations. This is the commonly used method due to its simplicity regarding parameters identification. This fitting is equivalent to a step by step minimization problem. Each step handles one parameter while the others are fixed. However, if the number of parameters increases, the minimization problem becomes more complex. Additionally, in case of enrichment of one of the studied parameters, the whole fitting have to be updated. Thus, this method becomes less efficient and numerically costly. Therefore, in this work, an optimization method was implemented to simplify the process of model parameters' regression. First, a database of explicit simulations on RVE was built to cover the space of dimensions (mechanical $\bar{\epsilon}, T_{x}, \mu$ and geometrical $\left.p_{i}, \gamma_{i}\right)$. This database can contain a very large number of simulations, all information coming from the Forge ${ }^{\circledR}$ software outputs $\left(V / V_{0}, T_{x}, \mu, \bar{\sigma}, \dot{\bar{\epsilon}}, \ldots\right)$ are stored and can be easily enriched if new simulations are necessary. An analytical function is defined by the user to represent the model. This analytical function contains constants $\left(A_{i}\right)$ which must be determined using inverse analysis methods. All types of functions could be used. For example, the following equation, presenting a polynomial form containing all parameters, can be used:

$$
\begin{aligned}
\frac{V}{V_{0}}= & A_{0}+\left(A_{1} \cdot T_{x}+A_{2} \cdot \mu+A_{3} \cdot p_{1}+A_{4} \cdot \gamma_{1}+A_{5} \cdot p_{2}+A_{6} \cdot \gamma_{2}+A_{7} \cdot p_{3}+A_{8} \cdot \gamma_{3}\right) \cdot \bar{\epsilon}+\left(A_{9} \cdot T_{x}^{2}+\right. \\
& \left.A_{10} \cdot \mu^{2}+A_{11} \cdot p_{1}^{2}+A_{12} \cdot \gamma_{1}^{2}+A_{13} \cdot p_{2}^{2}+A_{14} \cdot \gamma_{2}^{2}+A_{15} \cdot p_{3}^{2}+A_{16} \cdot \gamma_{3}^{2}\right) \cdot \bar{\epsilon}^{2}+\ldots
\end{aligned}
$$

An oriented object library was coded to manage user-defined functions. In fact, this optimization library ("optimizer") is coupled with the simulations database. This coupling allows the application of inverse analysis techniques to find the constants associated with the analytical function defined by the user. These inverse analysis techniques are used to minimize the $L^{2}-$ norm of the error between the 
evolution of the volume of the RVE explicit simulation $\left(\frac{V}{V_{0}}\right)^{\text {num }}$ and predicted volume given by the meanfield model $\left(\frac{V}{V_{0}}\right)^{\text {mod }}$, as expressed in Eq. (17).

$$
f=\min \left\|\left(\frac{V}{V_{0}}\right)^{\text {num }}-\left(\frac{V}{V_{0}}\right)^{\bmod }\right\|_{L^{2}} .
$$

The advantage of this method lies in its flexibility. Indeed, one can easily test different models (analytical forms) and thus assess their relevance. Testing a new model just requires the modification of the selected analytical function and a new otimization stage based on the datababase which already exists. It is worth mentioning that the optimization is performed until the critical value $V / V_{0}=0.2$. The reason for this is the change of slope observed in the last stages of closure (Figures $6 \& 7$ ). It was also mentionned by (Saby et al., 2015) that this issue is due to the contact between void surfaces and depends on voids tortuosity of the void. In case of equivalent geometries, the importance of this change of slope depends on the shape and orientation of the void (Figure 7). For real industrial voids, this change is more severe and depends a lot on voids tortuosity (Saby, 2013). Consequently, only the interval $V / V_{0} \in[0.2,1]$ will be considered for identification. Thereafter, the model will be compared with explicit simulations until the critical value $V / V_{0}=0.2$.

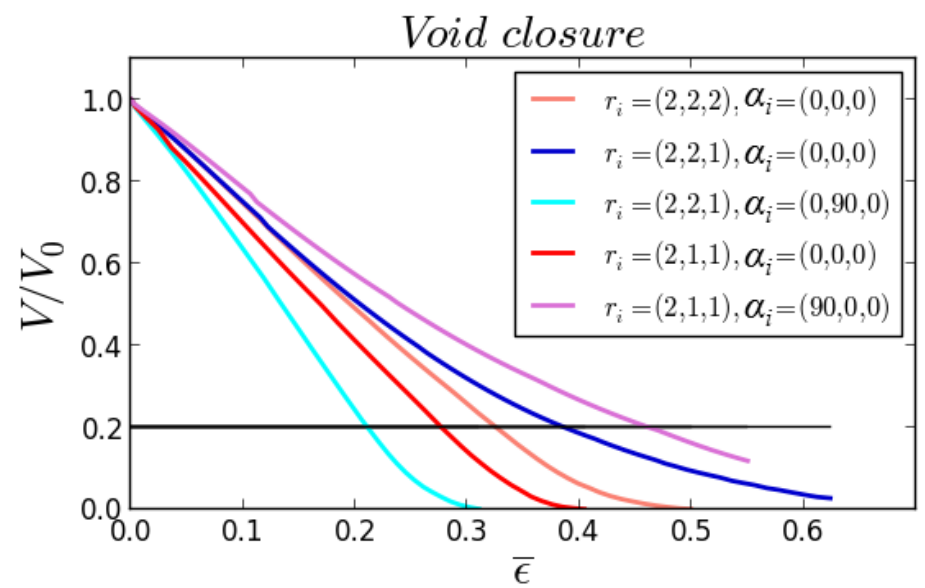

Figure 7: The influence of void's shape and orientation on void closure, for $T_{x}=$ $-0.33, \mu=1$. 


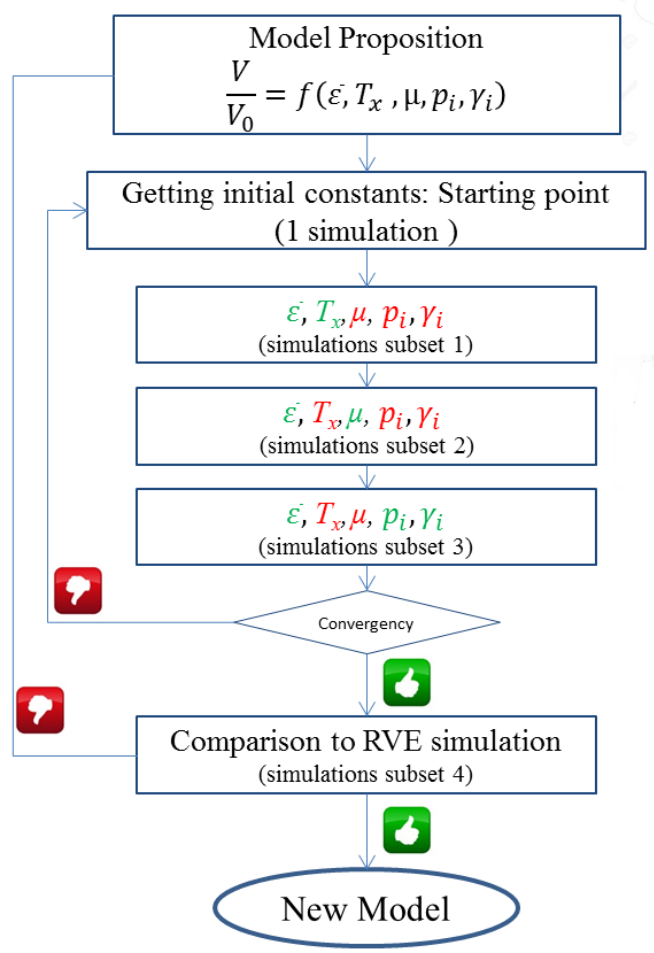

Figure 8: Main steps of the optimization algorithm used to develop the mean field model.

The optimization algorithm is composed of three main steps as illustrated in Figure 8. The first step consists in manually providing a mathematical formula that defines the model taking into account all the parameters that influence the evolution of closure, namely: $\bar{\epsilon}, T_{x}, \mu, \gamma_{i}$ and $p_{i}$. Due to the parabolic form observed in the evolution of void closure for different orientations and forms (Figure 7) and for different mechanical loading conditions (Figure 6), a second order polynomial function is proposed, see Eq. (18). According to the form of RVE explicit simulation evolution of void closure, a quadratic dependance on equivalent strain $\bar{\epsilon}$ is chosen. The analytical function presents also a quadratic dependance to the other parameters as detailed in Eq. (18). As it was presented in the general analytical equation (see in Eq. (16)), six constants correspond to void's dimension and six constants correspond to orientation properties, which is too weighty regarding the minimization 
problem. Consequently, in order to reduce the number of model constants, as the Lode parameter $\mu$ is also studied here, only two constants are assigned to $S$ and $S_{c}$ which represents a combination of void topology parameters as it is mentionned in Eq. (18).

$$
\frac{V}{V_{0}}=1+\left(A_{0}+A_{1} T_{x}+A_{2} \mu+A_{3} S\right) \bar{\epsilon}+\left(A_{4}+A_{5} T_{x}^{2}+A_{6} \mu^{2}+A_{7} S_{c}\right) \bar{\epsilon}^{2},
$$

with: $S=\sum_{i=1}^{3} \gamma_{i} \cdot p_{i}$ and $S_{c}=\sum_{i=1}^{3}\left(\gamma_{i} \cdot p_{i}\right)^{2}$. Where $A_{i}$ are the fitting constants, $p_{i}$ are the orientation factors and $\gamma_{i}$ are the shape factors.

\subsection{The model calibration procedure}

The model calibration procedure is illustrated in Figure 8. The Broyden-FletcherGoldfarb-Shanno algorithm (BFGS), which is an iterative method commonly used for solving nonlinear optimization problems, is used within the optimizer to solve the minimization problem (Nazareth, 1979). Thus, a starting point is required. In order to obtain fast convergence, the initial set of constants should be as close as possible to the optimal set of constants. For this reason, a first optimization step over a single RVE simulation is carried out. Using only one simulation, randomly chosen, this step enables to find a starting point very quickly. The result of this initial fitting step is plotted in Figure 9. These constants are then used as initial constants for the second stage of optimization.

The second step consists in a loop which contains three optimization iterations (Figure 8). In these three iterations, the various constants of the model are identified sequentially, the red parameters are fixed while green ones change. Table 1 presents the different sets used for these optimization. Note that for each set, green parameters will vary in the identified range, while the fixed ones are randomly chosen in the database. The first iteration concerns the variation of stress triaxiality ratio $T_{x}$ (set 1). The second iteration is for Lode parameter $\mu$ (set 2). The third one is run for the variation of void's morphology and orientation $\left(\gamma_{i} \& p_{i}\right)$ (set 3 ). Note that in 


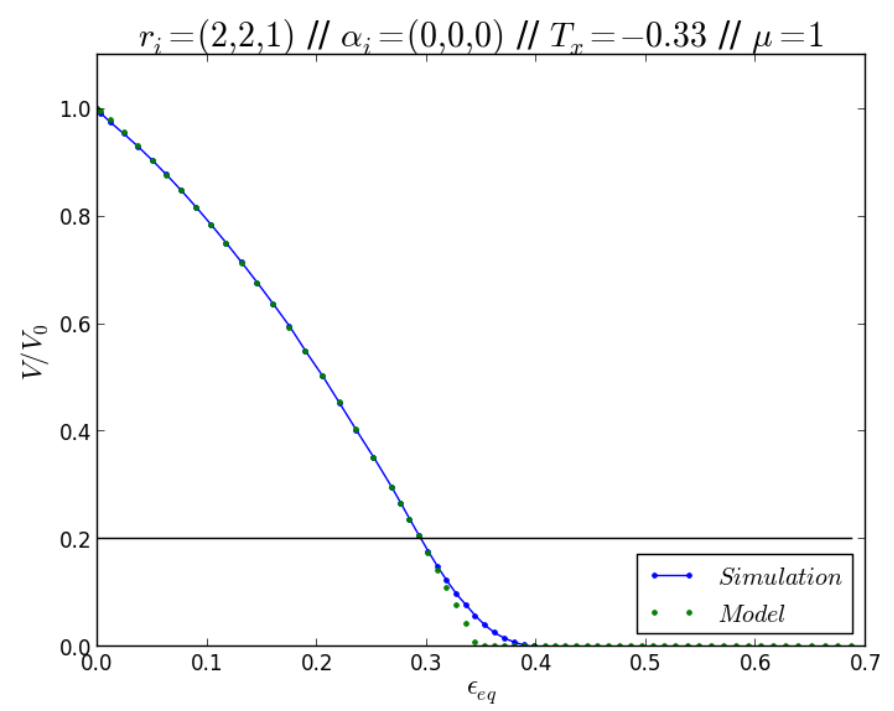

Figure 9: Optimization results to get initial conditions for the loop.

this set, four ellipsoid configurations are used to study shape influence. Concerning the orientation, every ellipsoid case represents a combination of three rotations $\alpha_{1}, \alpha_{2}, \alpha_{3}$, in the three directions $\overrightarrow{e_{1}}, \overrightarrow{e_{2}}, \overrightarrow{e_{3}}$ in the range $\left[0^{\circ}, 90^{\circ}\right]$ as mentionned in set 3.

For instance, set 1 of simulations, where $T_{x}$ is varied while $\mu, p_{i}$ and $\gamma_{i}$ are fixed, is used to fit the constants $A_{0}, A_{1}, A_{4}$ and $A_{5}$ while $A_{2}, A_{3}, A_{6}$ and $A_{7}$ are considered as fixed parameters. These parameters are assigned thanks to the previous step as explained in (Figure. 10). Each iteration step uses a different set of simulations (set 1 , set 2 or set 3 ) and stops when the optimizer reaches a very low error, defined by the user, with respect to void volume decrease coming from the database. The set of simulations is chosen so as to give more weight to the modified parameters: $T_{x}$ for set $1, \mu$ for set 2 and $\left(p_{i} \& \gamma_{i}\right)$ for set 3 .

On entering the loop, the three optimization iterations will be repeated until the convergence condition is satisfied. Convergence is reached when the distance between two solutions $k$ and $k-1$ (as defined in Eq. (19)) is lower than a threshold specified by the user.

$$
\operatorname{cvg}=\frac{\left\|A_{i}^{k}-A_{i}^{k-1}\right\|_{L^{2}}}{\left\|A_{i}^{k-1}\right\|_{L^{2}}} .
$$




\begin{tabular}{|c|c|c|c|c|}
\hline \hline Varied parameters & $T_{x}$ & $\mu$ & Dimensions $r_{i}$ & orientation $\alpha_{i}$ \\
\hline \hline $\begin{array}{c}\text { Set 1 } \\
\text { Variation of } T_{x}\end{array}$ & {$[-1,0]$} & 1 & $(2.5,1.8,1.1)$ & $\left(45^{\circ}, 60^{\circ}, 75^{\circ}\right)$ \\
\hline Set 2 & -0.33 & {$[0,1]$} & $(2.5,1.8,1.1)$ & $\left(45^{\circ}, 60^{\circ}, 75^{\circ}\right)$ \\
Variation of $\mu$ & & & $(2.5,1.8,1.1)$ & \\
\hline & & & $(2,1,1)$ & $(2,2,1)$ \\
Set 3 & -0.33 & 1 & $(3.2,2.2,1.4)$ & \\
\hline Variation of $r_{i} \& \alpha_{i}$ & & & \multicolumn{3}{c}{} \\
\hline
\end{tabular}

Table 1: Used configurations for mean field model calibration.

$$
\frac{V}{V_{0}}=1+\left(A_{0}+A_{1} \cdot T_{x}+A_{2} \cdot \mu+A_{3} \cdot S\right) \cdot \bar{\varepsilon}+\left(A_{4}+A_{5} \cdot T_{x}^{2}+A_{6} \cdot \mu^{2}+A_{7} \cdot S_{c}\right) \cdot \bar{\varepsilon}^{2}
$$

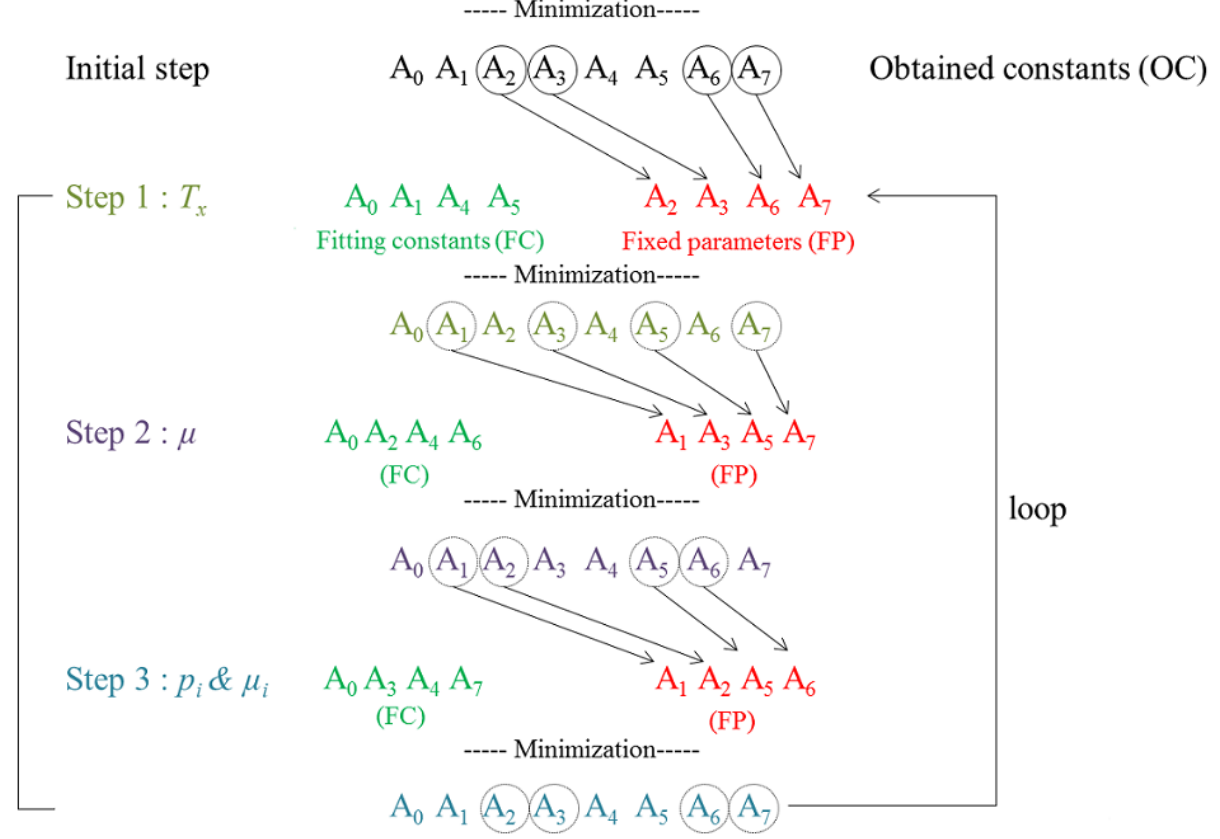

Figure 10: Parameters and constants assignment in the optimization loop.

\section{Validation of the mean field model}

\section{1 comparison with explicit simulations}

In order to validate the new mean field model for void closure with the effect of Lode 22

angle, the predictions are compared with a large number of explicit RVE simulations 
to show how the model can predict the void volume evolution for different void's orientations and shapes and for different mechanical loading conditions. A total of ninety cases are compared (Table 2), four of these cases are represented in Figure 11. The void volume evolution is plotted with respect to equivalent strain. The new mean field model is plotted with red dots while the explicit simulation is plotted with a solid black line. The straight line $y=0.2$ represents the critical value of void closure. The value $E_{r}$ (in the legend) represents the mean error (Eq. 20), between the new model $\left(\frac{V}{V_{0}}\right)_{\text {mod }}$ and explicit RVE simulations $\left(\frac{V}{V_{0}}\right)_{\text {num }}$ over the whole range of strain. The title above each subfigure contains informations about the simulation, $r_{i}$ represents the initial ellipsoid's dimensions, $\alpha_{i}$ represents the initial orientations of the ellipsoid in the RVE. Thus, the void's shape factors $\gamma_{i}$ and orientations factors $p_{i}$ are calculated as detailed in Eq. (12) and Eq. (14) using $\alpha_{i}$ and $r_{i}$, respectively.

$$
E_{r}=\frac{1}{N} \sum_{i}^{N}\left|\left(\frac{V_{i}}{V_{0}}\right)_{\text {num }}-\left(\frac{V_{i}}{V_{0}}\right)_{\text {mod }}\right| .
$$

Where $N$ is the number of points in the range of strain.

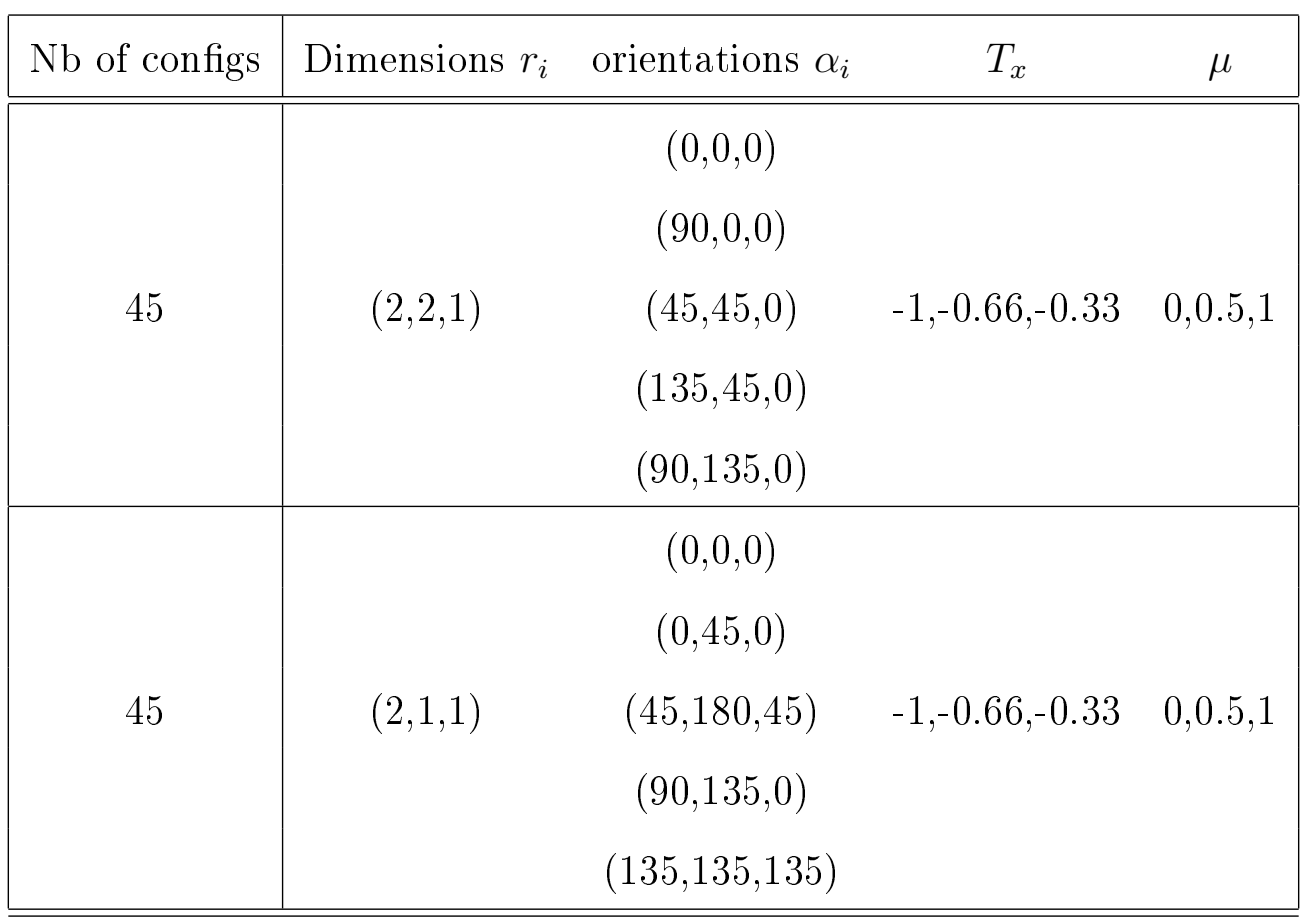

Table 2: Different configurations used for comparison with explicit simulations. 
As it is shown in Figure 11, all presented cases are well predicted, with small discrepancies. In the interval [0,0.2], a change of slope is observed in the curve of the explicit simulation. This phenomenon causes more serious discrepancies as the model is defined by a quadratic form, and therefore cannot reproduce this change of slope. However, the results are promising and the prediction is very accurate in the range $\bar{\epsilon} \in[0.2,1]$, especially for axisymmetric loading $(\mu=1)$.

The mean error (Eq. 20) is calculated for all cases and does not exceed $14 \%$. The maximal error corresponds to a case of orientation which could be considered as an extreme case because the ellipsoid's largest dimension is aligned with the deformation direction which is not desirebale for closure. In addition, the error average is $3.29 \%$ while the standard deviation is equal to $2.49 \%$ which indicates that error points are clustered closely around the average. As it is seen in Figure 12, the error of $85.56 \%$ cases is less than $5.78 \%$ which corresponds to the sum of the average $\overline{E_{r}}$ and the standard deviation $\sigma\left(\overline{E_{r}}+\sigma\right)$. In statistics, the Poisson distribution is usually used to study the rare events. In order to apply the Poisson distribution, some conditions have to be satisfied: (i) the occurence of events is independent, (ii) events occur with constant density $\lambda$, and (iii) the event is something that can be counted in integers (Grais, 2003) and (Whittle, 1970). Let us consider the error vector for the ninety simulations used for comparison as a random variable. The errors will be truncated in order to have integers. Consequently, the error distribution could be approximated by a Poisson distribution as mentionned with red solid line in Figure 12 with $\lambda=3$ which is the average and the variance (truncated) of the error distribution.

\subsection{Comparison with the Cicaporo1 model}

After validating the model with RVE explicit simulations, this paragraph compares the results with the model Cicaporo1 which was defined for axisymmetric loading conditions (Saby et al., 2014b) expressed as: 


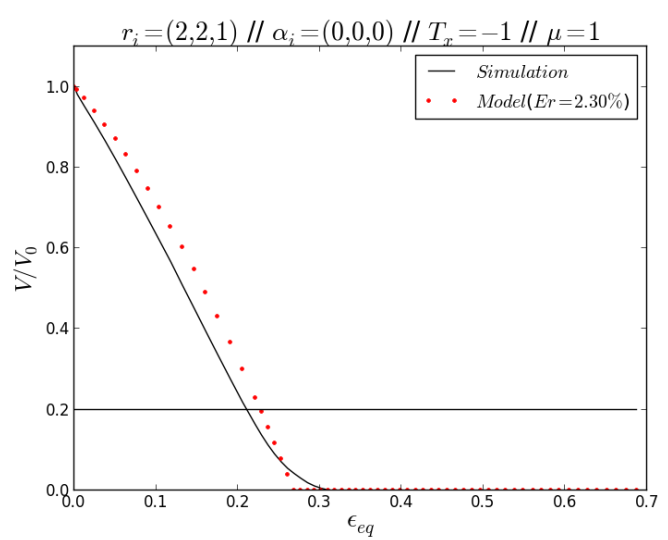

(a)

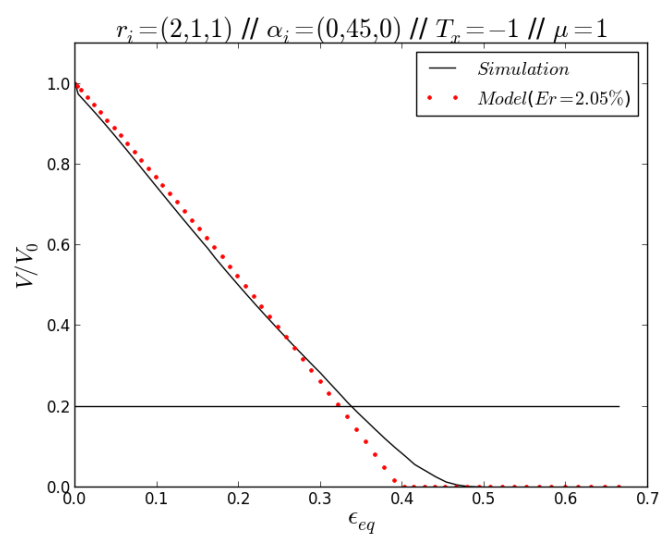

(c)

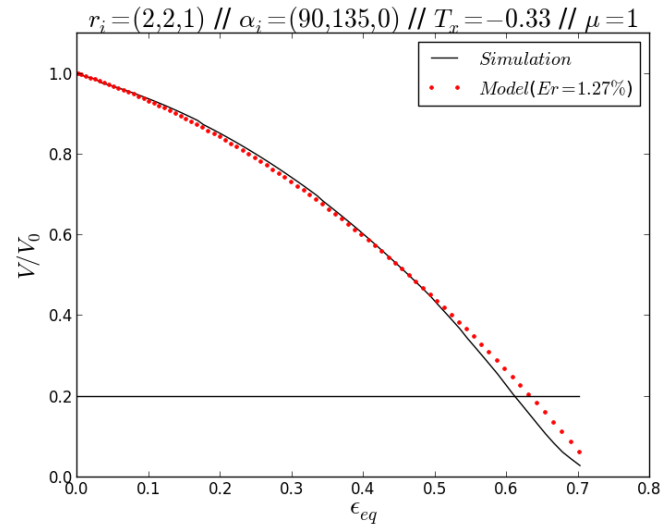

(b)

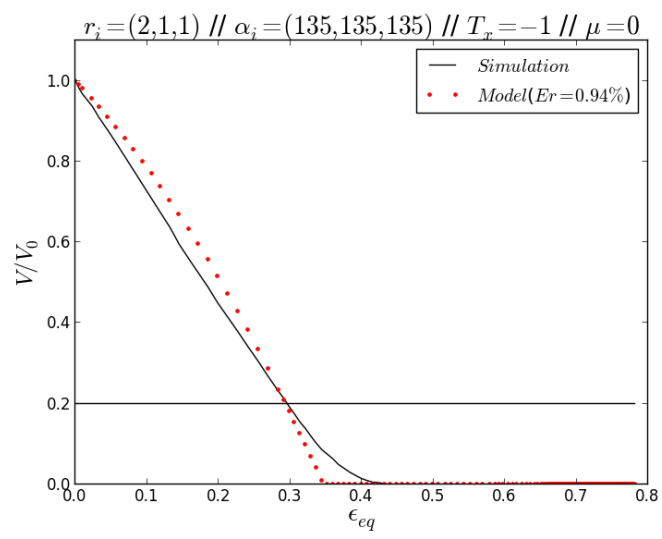

(d)

Figure 11: Comparison between the new model and explicit RVE simulation for different void configurations (shape \& orientations) and mechanical loadings $\left(T_{x} \&\right.$ $\mu)$.

$$
\frac{V}{V_{0}}=1+B \bar{\epsilon}+C \bar{\epsilon}^{2}
$$

with

$$
\begin{aligned}
B & =\sum_{i=1}^{3} \sum_{j=0}^{2} \sum_{k=0}^{1} b_{j k}\left(T_{x}\right)^{k}\left(\gamma_{i}\right)^{j} p_{i}, \\
C & =\sum_{i=1}^{3} \sum_{j=0}^{2} \sum_{k=0}^{2} c_{j k}\left(T_{x}\right)^{k}\left(\gamma_{i}\right)^{j} p_{i},
\end{aligned}
$$

where the numerical values of six constants $b_{j k}$ and the nine constants $c_{j k}$ are fitted using linear regression. 


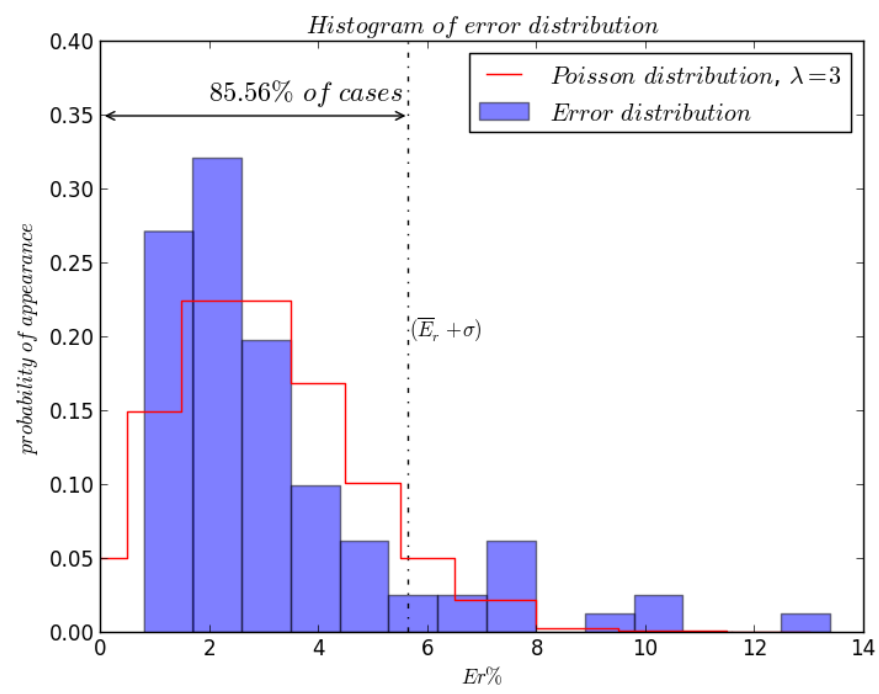

Figure 12: Histogram of the probability of the mean error of the new model prediction with the Poisson distribution $(\lambda=3)$.

\subsubsection{For axisymmetric loading conditions}

Different configurations are studied for different states of triaxiality and for $\mu=1$, because the Cicaporo1 was calibrated for axisymmetric loading conditions only. In Figure 13, explicit RVE simulations are plotted with solid lines, the Cicaporo2 model and CicaPoro1 model are plotted with dots and stars, respectively.

In Figures 13, both models present good accuracy. The error $E r_{0.2}$ (see Eq. 22), which is the relative difference between model and explicit RVE simulation at the critical value of closure $V / V_{0}=0.2$, is lower than $5.11 \%$. Table 3 presents errors of both models regarding explicit RVE simulations, thanks to Eq. (22), for the presented axisymmetric loading configurations. For the thirty cases studied, the prediction given by the Cicaporo2 model is very good. The error $E r_{0.2}$ does not exceed $15 \%$.

$$
E r_{0.2} \%=\frac{\bar{\epsilon}_{\text {sim }}-\bar{\epsilon}_{\text {mod }}}{\bar{\epsilon}_{\text {sim }}} * 100
$$

with $\bar{\epsilon}_{s i m}$ and $\bar{\epsilon}_{m o d}$ are the equivalent strains of the explicit RVE simulation and the predicted model respectively for $\frac{V}{V_{0}}=0.2$. 


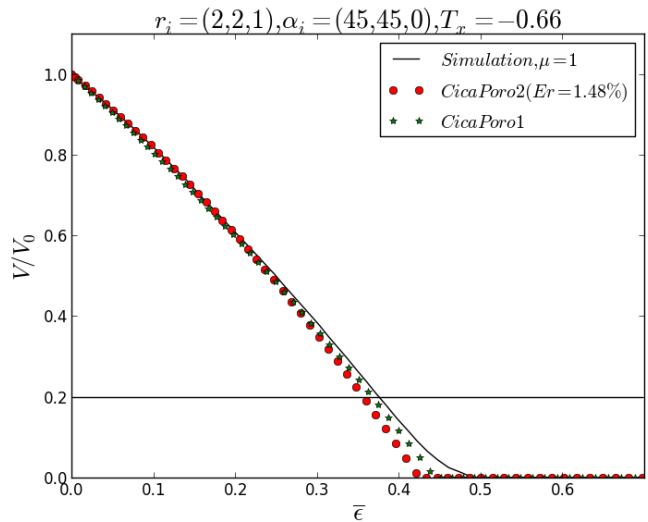

(a) $T_{x}=-0.66$

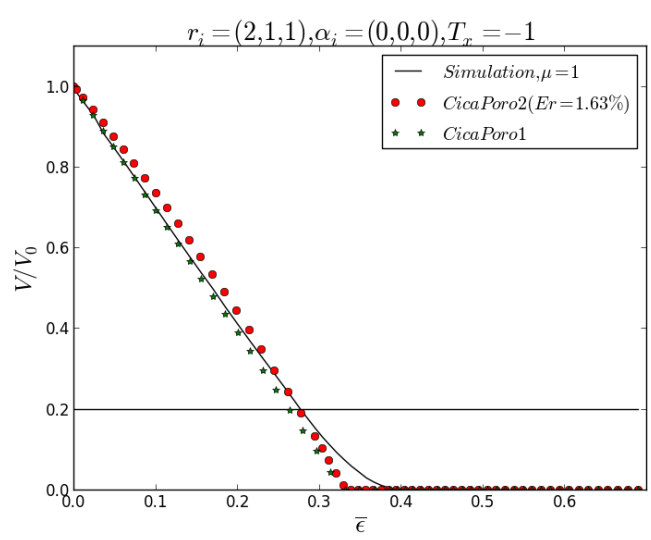

(c) $T_{x}=-0.66$

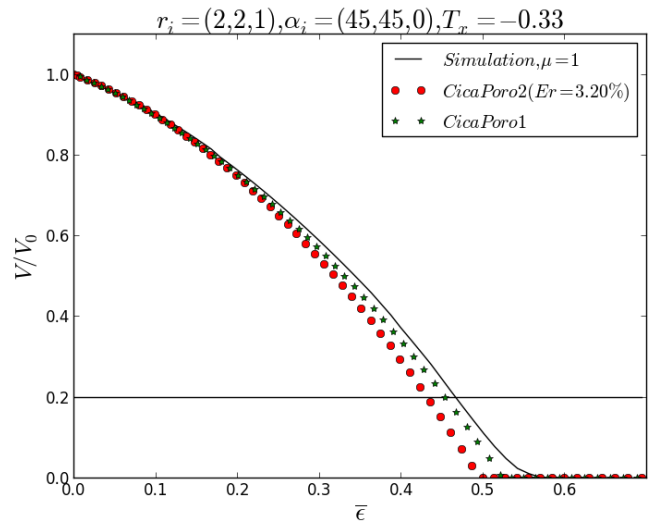

(b) $T_{x}=-0.33$

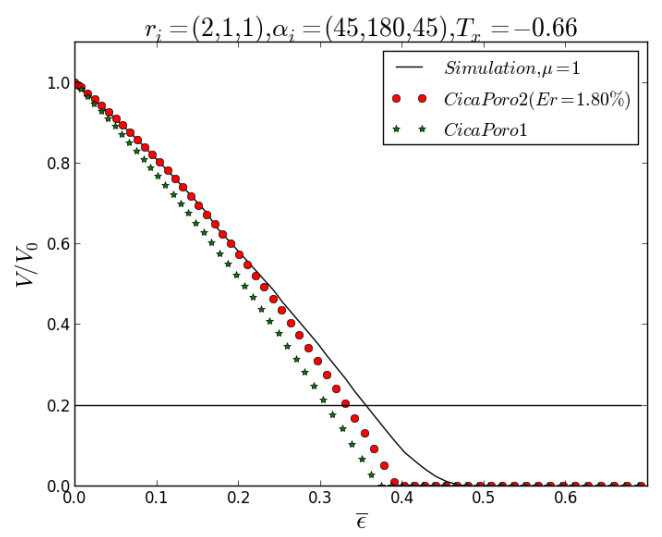

(d) $T_{x}=-1$

Figure 13: Comparison between Cicaporo2 model, explicit RVE simulation and Cicaporo1 model for four different void configurations (shape \& orientations) and mechanical loadings.

\subsubsection{For general stress states}

In order to show the improvement of the new Cicaporo 2 model compared to the Cicaporo1 formulation, comparisons are now made with more general stress states (Lode parameter equal to $0,0.5$ and 1). The error $E r_{0.2}$ (Eq. 22) is considered for this comparison. In Table 4, values of the error $E r_{0.2}$ are presented for both Cicaporo2 and Cicaporo1 models for $\mu=1, \mu=0.5$ and $\mu=0$. Note that for Cicaporo1 model, the value $\bar{\epsilon}_{m o d}$ is the same for the three cases of $\mu$ since the model is calibrated for $\mu=1$. 


\begin{tabular}{|c|c|c|c|c|}
\hline$\mu$ & Configurations & $T_{x}$ & $\begin{array}{c}E r_{0.2} \% \\
\text { Cicaporo2 }\end{array}$ & $\begin{array}{c}E r_{0.2} \% \\
\text { Cicaporo1 }\end{array}$ \\
\hline \hline \multirow{3}{*}{1} & \multirow{2}{*}{$r_{i}=(2,2,1), \alpha_{i}=(45,45,0)$} & -0.33 & 5.06 & 2.12 \\
\cline { 3 - 5 } & \multirow{2}{*}{$r_{i}=(2,1,1), \alpha_{i}=(0,0,0)$} & -0.66 & 5.06 & 2.12 \\
\cline { 3 - 5 } & & -0.33 & 2.15 & 2.89 \\
\cline { 3 - 5 } & & -0.66 & 0.82 & 1.16 \\
\hline
\end{tabular}

Table 3: Comparison between Cicaporo2 and Cicaporo1 models with explicit RVE simulations for axisymmetric loading conditions using the expression Eq. (22).

In Figure 14, the comparison between four cases is presented. Explicit RVE simulations are plotted with solid lines. Cicaporo1 and Cicaporo2 are plotted with stars and dots respectively. The cases $\mu=1, \mu=0.5$ and $\mu=0$ are plotted with red, blue and green, respectively. Note that Cicaporo1 is plotted only for $\mu=1$, the condition for which it was calibrated. However, it is compared with explicit simulations for other cases to evaluate its discrepancy for other stress states. In the first case (Figure 14a), the prediction is acceptable for the Cicaporo2 model, especially in the range $[0.2,1]$ of void closure. The error $e_{0.2}$ is reduced from $7.59 \%$ for Cicaporo 1 to $3.32 \%$ for Cicaporo 2 model for the case $\mu=1$. For non-axisymmetric loading conditions, the Cicaporo 2 model errors are equal to $8.33 \%$ and $10.31 \%$ for the cases $\mu=0.5$ and $\mu=0$ respectively while the Cicaporo1 model errors are $13.3 \%$ and $22.68 \%$ respectively. Even though the model is meant to be used for $\mu=1$, if it were compared to other loading, the error would be much higher. In table 4, errors of all presented cases are detailed.

For the presented cases, as for the all tested configurations, the case $\mu=0$ represents the biggest discrepancy, while the case $\mu=1$ always leads the best prediction. This could be due to the chosen simulations for the calibration process. Indeed, all explicit RVE simulations used for constants identification when Lode parameter $\mu$ is fixed, $\mu$ is equal to 1 . In order to investigate this point and capitalize on the 


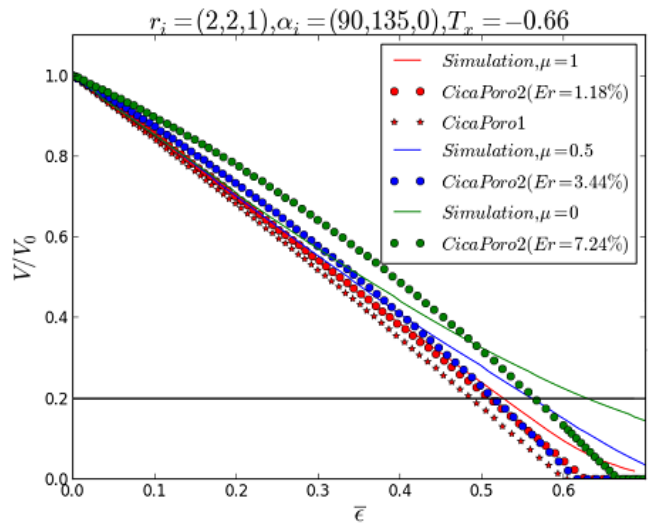

(a)

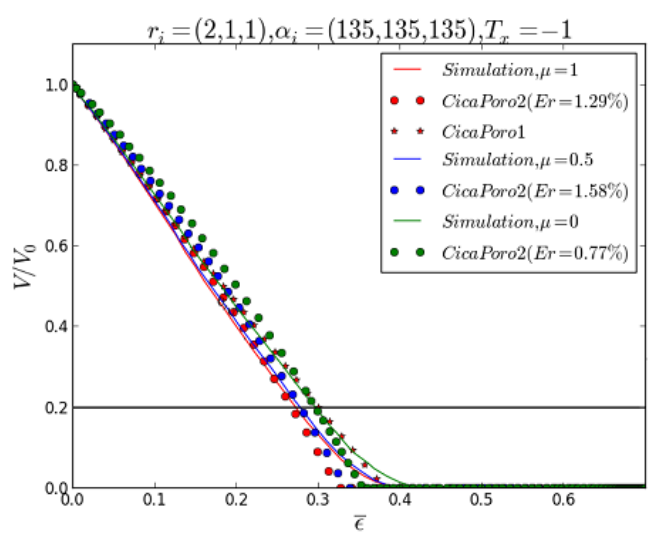

(c)

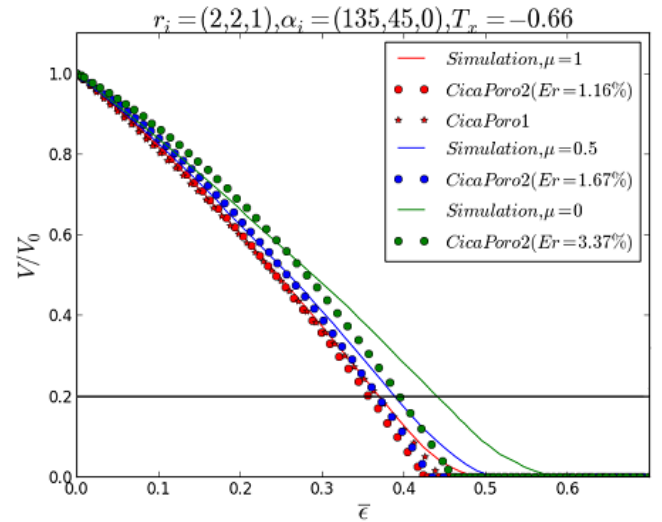

(b)

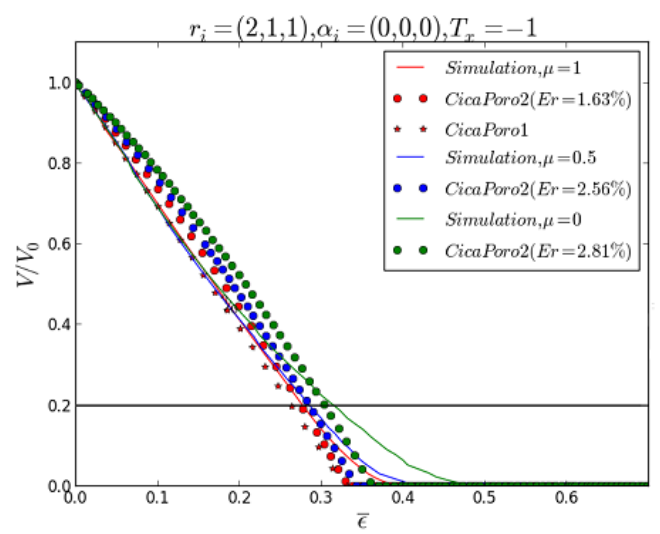

(d)

Figure 14: Comparison between the Cicaporo2 model and Cicaporo1 model with explicit RVE simulation for different void configurations (shape \& orientations) and mechanical loadings.

optimization procedure flexibility, a database enrichment was made and results are presented in the next section.

\subsubsection{Influence of the set of explicit RVE simulations chosen for the calibration stage}

A new set of explicit RVE simulations was used to replace the set 2 (see Table 1) which handle the variation of $T_{x}$. Lode parameter is now fixed to 0 . The optimization was run and an improvment of the mean error (Eq. 20) is stated. In fact, the maximal error between the explicit RVE simulation and the prediction of Cicaporo2 


\begin{tabular}{|c|c|c|c|c|c|c|c|c|}
\hline \multirow{2}{*}{ Configurations } & \multirow{2}{*}{$T_{x}$} & \multicolumn{6}{|c|}{$E r_{0.2} \%$} \\
\cline { 5 - 8 } & & & \multicolumn{2}{|c|}{$\mu=1$} & \multicolumn{2}{c|}{$\mu=0.5$} & \multicolumn{2}{c|}{$\mu=0$} \\
\cline { 5 - 9 }$r_{i}$ & $\alpha_{i}$ & & Cica2 & Cica1 & Cica2 & Cica1 & Cica2 & Cica1 \\
\hline \hline$(2,2,1)$ & $(90,135,0)$ & -0.66 & 3.32 & 7.59 & 8.33 & 13.3 & 10.31 & 22.68 \\
\hline$(2,2,1)$ & $(135,45,0)$ & -0.66 & 3.42 & 0.42 & 5.28 & 5.31 & 10.51 & 16.45 \\
\hline$(2,1,1)$ & $(135,135,135)$ & -1 & 1.73 & 10.34 & 0.12 & 8.16 & 0.06 & 1.42 \\
\hline$(2,1,1)$ & $(0,0,0)$ & -1 & 0.82 & 5.11 & 0.00 & 7.90 & 3.5 & 16.63 \\
\hline \hline
\end{tabular}

Table 4: Comparison between Cicaporo2 and Cicaporo1 models error $\operatorname{Er}_{0.2} \%$ (Eq. 22) for general stress states.

model decreases to $10.76 \%$ instead of $13.39 \%$ for $\mu=1$ in set 2 . The average of the error remains almost the same with $3.47 \%$ instead of $3.29 \%$. This distribution can also be approximated by a Poisson law with $\lambda=3$ (see Figure 15 .

Consequetly, one can confirm that the model is a case-dependent as long as the optimization result changes within the change of the sets of explicit RVE simulation used for model calibration. However, the solution given by the second optimization is close to the first one: $\frac{\left\|A_{i}^{\mu=1}-A_{i}^{\mu=0}\right\|_{L^{2}}}{\left\|A_{i}^{\mu=1}\right\|_{L^{2}}}=0.20$, with $A_{i}^{\mu=1}$ and $A_{i}^{\mu=0}$ are the model constants given by the first ( $\mu=1$ in set 2$)$ and second ( $\mu=0$ in set 2 ) optimizations, respectively.

\section{Conclusions}

The Lode angle was investigated in order to study void closure for more general stress states to avoid the hypothesis of axisymmetric loading always used to study void closure in the literature. Its influence on void volume evolution was demonstrated and two conclusions were raisen: 1- the axisymmetric loading $(\mu=1)$ represents the most favourable case of closure, 2 - the higher is $T_{x}$ (in our interval of interest), the more important is the use of $\mu$ in void closure models. 


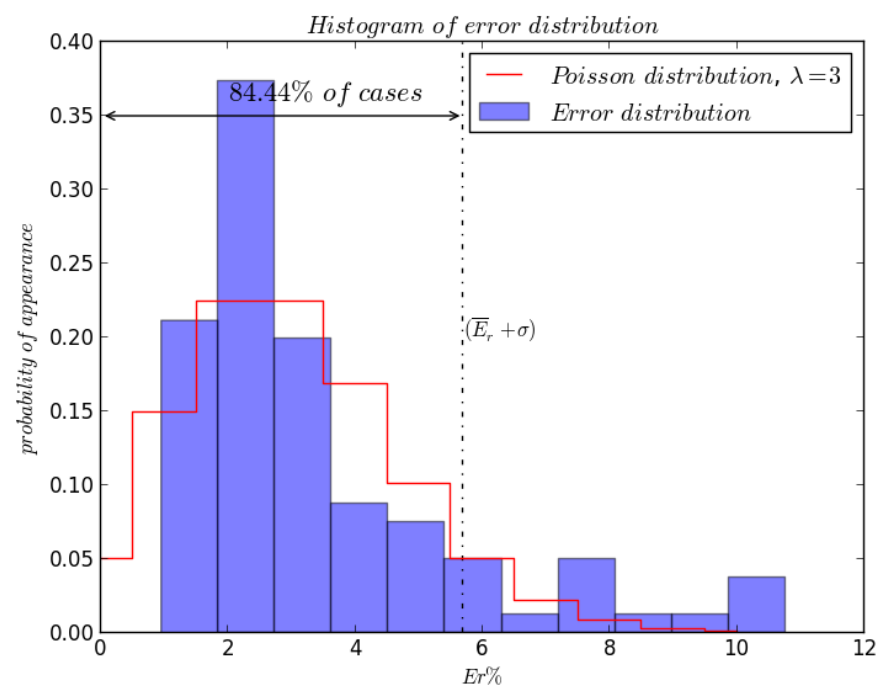

Figure 15: Histogram of the error and Poisson law distribution with the new optimization.

An optimization method using a database has been developed. This methodology allows to easily test new mathematical forms of void closure model. New simulations coming from the database can be added to the set of simulations used for calibration which makes this method very efficient. This methodology was used here to calibrate the Cicaporo2 model taking into account void's morphology (shape \& orientation) and mechanical loading conditions (stress triaxiality ratio $T_{x} \&$ Lode parameter $\mu)$. The model was validated by comparing model predictions with explicit RVE simulations. Ninety configurations were analyzed and a good accuracy was obtained for all of them. For axisymmetric loading conditions, Cicaporo2 model predicts void volume evolution as well as the Cicaporo1 model (which was already considered as an accurate prediction model for void closure for axisymmetric loading conditions). The most important result is obtained for the non axisymmetric cases, where the accuracy is significantly improved compared with Cicaporo1 model. It is expected that the Cicaporo2 model would also give better prediction than any other mean field model from the literature for non axisymmetric loading conditions since this is the first model calibrated for general stress states and accounting for the Lode angle 
dependance.

Further work will be dedicated to a tensorial form of the model which could predict void shape evolution in addition to void volume closure. This is necessary since void closure is achieved through multi-stages forming operations in the industrial processes where the workpiece is usually rotated between each forming stage. As the shape and orientation of voids are first order parameters regarding void closure, it is essential to keep track of these parameters during all multistages void closure processes.

\section{Acknowledgement}

The authors would like to gratefully thank ArcelorMittal Gandrange \& Industeel, Areva TN, Asco Industries, Aubert\&Duval, Constellium CRV and Timet Savoie for funding this research. 


\section{References}

B Budiansky, J-W Hutchinson, and S Slutsky. Void growth and collapse in viscous solids. pages 13-45, 1982.

T-S Cao, E Maire, C Verdu, C Bobadilla, P Lasne, P Montmitonnet, and P-O Bouchard. Characterization of ductile damage for a high carbon steel using $3 \mathrm{~d}$ x-ray micro-tomography and mechanical tests-application to the identification of a shear modified gtn model. Computational Materials Science, 84:175-187, 2014.

K Danas and P-P Castañeda. Influence of the lode parameter and the stress triaxiality on the failure of elasto-plastic porous materials. International Journal of Solids and Structures, 49(11):1325-1342, 2012.

C Feng and Z Cui. A 3-d model for void evolution in viscous materials under large compressive deformation. International Journal of Plasticity, 74:192-212, 2015.

B Grais. Methodes statistiques. Dunod, 2003.

H Kakimoto, T Arikawa, Y Takahashi, T Tanaka, and Y Imaida. Development of forging process design to close internal voids. Journal of Materials Processing Technology, 210(3):415-422, 2010.

A Keshavarz, R Ghajar, and G Mirone. A new experimental failure model based on triaxiality factor and lode angle for x-100 pipeline steel. International Journal of Mechanical Sciences, 80:175-182, 2014.

B-J Lee and M-E Mear. Studies of the growth and collapse of voids in viscous solids. Journal of engineering materials and technology, 116(3):348-358, 1994.

W Lode. Versuche über den einfluß der mittleren hauptspannung auf das fließen der metalle eisen, kupfer und nickel. Zeitschrift für Physik, 36(11-12):913-939, 1926.

G Mirone and D Corallo. A local viewpoint for evaluating the influence of stress 
triaxiality and lode angle on ductile failure and hardening. International Journal of Plasticity, 26(3):348-371, 2010.

L Nazareth. A relationship between the bfgs and conjugate gradient algorithms and its implications for new algorithms. SIAM Journal on Numerical Analysis, 16(5): 794-800, 1979.

C-Y Park and D-Y Yang. A study of void crushing in large forgings i: Bonding mechanism and estimation model for bonding efficiency. Journal of materials processing technology, 57(1):129-140, 1996.

M Saby. Understanding and modeling of void closure mechanisms in hot metal forming processes. PhD thesis, 2013.

M Saby, M Bernacki, E Roux, and P-O Bouchard. Three-dimensional analysis of real void closure at the meso-scale during hot metal forming processes. Computational Materials Science, 77:194-201, 2013.

M Saby, M Bernacki, and P-O Bouchard. Understanding and modeling of void closure mechanisms in hot metal forming processes: a multiscale approach. Procedia Engineering, 81:137-142, 2014a.

M Saby, P-O Bouchard, and M Bernacki. Void closure criteria for hot metal forming: a review. Journal of Manufacturing Processes, pages 239-250, 2014 b.

M Saby, P-O Bouchard, and M Bernacki. A geometry-dependent model for void closure in hot metal forming processes. Finite Elements in Analysis and Design, pages $63-75,2015$.

U Ståhlberg, H Keife, M Lundberg, and A Melander. A study of void closure during plastic deformation. Journal of Mechanical Working Technology, 4(1):51-63, 1980.

M Tanaka, S Ono, and M Tsuneno. Factors contributing to crushing of voids during forging. J. Jpn. Soci. Technol. Plast, 27(306):927-934, 1986. 
A Wallerö. Closing of a central longitudinal pore in hot rolling. Journal of Mechanical Working Technology, 12(2):233-242, 1985.

A Wang, PF Thomson, and PD Hodgson. A study of pore closure and welding in hot rolling process. Journal of Materials Processing Technology, 60(1):95-102, 1996.

P Whittle. Probability. Penguin Education, 1970.

L Xue and T Wierzbicki. Ductile fracture initiation and propagation modeling using damage plasticity theory. Engineering Fracture Mechanics, 75(11):32763293, 2008.

X-X Zhang, Z-S Cui, W Chen, and Y Li. A criterion for void closure in large ingots during hot forging. Journal of Materials Processing Technology, 209(4):1950-1959, 2009 . 